\title{
Buchi's Problem for ultrametric meromorphic functions inside an open disk
}

\author{
Alain Escassut, Hector Pasten
}

\begin{abstract}
Let $K$ be a complete ultrametric algebraically closed field of characteristic zero such as $\mathbb{C}_{p}$. Büchi's problem was solved for $p$-adic meromorphic functions in the whole field $K$. Here we show similar conclusions for meromorphic functions in an open disk that are not quotients of bounded analytic functions. The main method is the second Main Theorem for $p$-adic meromorphic functions inside a disk, a specific p-adic theorem.
\end{abstract}

2000 Mathematics subject classification: Primary 12J25 Secondary 46S10

\section{Introduction}

The purpose of this work is to prove the analogue of certain diophantine question known as Büchi's problem, over the field of non-archimedean meromorphic functions on an "open" disk. This problem for the ring of rational integers reads as follows

Problem 1.1 (Büchi's Problem). Does there exist some constant $M_{0}$ such that, if a sequence of square integers $x_{1}^{2}, x_{2}^{2}, \ldots, x_{n}^{2}$ has second differences constant and equal to 2 and $n \geq M_{0}$, then necessarily the $x_{i}^{2}$ are squares of consecutive integers?

The main motivation behind this problem came from Logic. Indeed, Büchi realized that a positive answer to this problem together with the negative answer to Hilbert's tenth problem given by Matijasevich, imply a very strong undecidability result about systems of diagonal quadratic equations.

If $x_{1}^{2}, \ldots, x_{n}^{2}$ have second differences equal to two, an easy argument shows that there exist integers $\nu, a$ such that $x_{k}^{2}=(k+\nu)^{2}-a$ for each $a$. Hence, one wants to find some $M_{0}$ with the property that, if $n \geq M_{0}$ then necessarily $a=0$.

A similar argument works in the context of fields of functions, but one has to consider as "trivial" the case where all the $x_{k}$ are constants. A very relevant case is the analogous problem for complex meromorphic functions, where Büchi's Problem was solved by Vojta in [?] and, in an equivalent formulation, he shows that if we are given $h_{1}, \ldots, h_{M}, f, g$ meromorphic functions over the whole $\mathbb{C}$ not all constant, such that

$$
h_{k}^{2}=(k+f)^{2}-g \quad \text { for each } j=1, \ldots, M
$$

then $M \leq 7$. Unfortunately, in contrast to the case of integers, this result has no undecidability consequence because Hilbert's tenth problem is an open problem for complex meromorphic functions (and even for rational functions over the complex numbers). In [?] and [?] the same problem was considered for fields of rational functions and polynomial rings in both characteristic zero and positive. It was natural to consider the same question for meromorphic and entire functions in a whole p-adic field (because in this case some analogues to Hilbert's tenth problem are known) and this was done in $[?]$.

Here we want to examine the same problem for ultrametric meromorphic functions defined in an "open" disk. One of the main reasons of why we can deal with this problem on open disks, is that 
in the non-archimedean setting Nevanlinna Theory works in a satisfactory way inside a disk, and for instance one gets results on the non-parametrization of hyper-elliptic curves [?], [?].

\section{Notation, definitions and main result}

Let $K$ be an algebraically closed field of characteristic 0 , complete with respect to an ultrametric absolute value $|\cdot|$. Given $\alpha \in K$ and $R \in \mathbb{R}_{+}^{*}$, we denote by $d(\alpha, R)$ the disk

$$
\{x \in K:|x-\alpha| \leq R\}
$$

and by $d\left(\alpha, R^{-}\right)$the disk

$$
\{x \in K:|x-\alpha|<R\},
$$

by $\mathcal{A}(K)$ the $K$-algebra of analytic functions in $K$ (i.e. the set of power series with an infinite radius of convergence) and by $\mathcal{M}(K)$ the field of meromorphic functions in $K$.

In the same way, given $\alpha \in K$ and $r>0$, we denote by $\mathcal{A}\left(d\left(\alpha, r^{-}\right)\right)$the $K$-algebra of analytic functions in $d\left(\alpha, r^{-}\right)$(i.e. the set of power series with a radius of convergence $\left.\geq r\right)$ and by $\mathcal{M}\left(d\left(\alpha, r^{-}\right)\right)$ the field of fractions of $\mathcal{A}\left(d\left(\alpha, r^{-}\right)\right)$. We then denote by $\mathcal{A}_{b}\left(d\left(\alpha, r^{-}\right)\right)$the $K$-algebra of bounded analytic functions in $d\left(\alpha, r^{-}\right)$and by $\mathcal{M}_{b}\left(d\left(\alpha, r^{-}\right)\right)$the field of fractions of $\mathcal{A}_{b}\left(d\left(\alpha, r^{-}\right)\right)$. And we set

$$
\mathcal{A}_{u}\left(d\left(\alpha, r^{-}\right)\right)=\mathcal{A}\left(d\left(\alpha, r^{-}\right)\right) \backslash \mathcal{A}_{b}\left(d\left(\alpha, r^{-}\right)\right)
$$

and

$$
\mathcal{M}_{u}\left(d\left(\alpha, r^{-}\right)\right)=\mathcal{M}\left(d\left(\alpha, r^{-}\right)\right) \backslash \mathcal{M}_{b}\left(d\left(\alpha, r^{-}\right)\right) .
$$

Throughout the paper, we denote by $\mathcal{D}$ a disk $d\left(a, R^{-}\right)$and by $M$ an integer $\geq 6$.

In [?] Buchi's problem for meromorphic functions was solved in the whole field:

Theorem 2.1. Let $f, g \in \mathcal{M}(K)$ with $g$ non-identically zero. Let $h_{1}, \ldots, h_{M} \in \mathcal{M}(K)$ with $h_{1}$ nonconstant and let $a_{1}, \ldots, a_{M} \in K$ be distinct and such that $h_{j}^{2}=\left(a_{j}+f\right)^{2}-g$ for each $j=1, \ldots, M$. Then $M \leq 34$. Moreover, if $h_{1}, \ldots, h_{M} \in \mathcal{A}(K)$, then $M \leq 12$

Here we want to show that this result also holds for meromorphic functions lying in $\mathcal{M}_{u}(\mathcal{D})$ :

Theorem 2.2. Let $f, g \in \mathcal{M}(\mathcal{D})$ with $g$ non-identically zero. Let $h_{1}, \ldots, h_{M} \in \mathcal{M}(\mathcal{D})$ with $h_{1} \in \mathcal{M}_{u}(\mathcal{D})$ and let $a_{1}, \ldots, a_{M} \in K$ be distinct and such that

$$
h_{j}^{2}=\left(a_{j}+f\right)^{2}-g
$$

for each $j=1, \ldots, M$. Then $M \leq 34$. Moreover, if $h_{1}, \ldots, h_{M} \in \mathcal{A}(D)$ with $h_{1} \in \mathcal{A}_{u}(\mathcal{D})$, then $M \leq 12$.

\section{Some preliminary results}

The proof of Theorem ?? requires several preliminary results. First, we must notice a very basic lemma:

Lemma 3.1. Let $E / F$ be a field extension and assume that $F$ is algebraically closed in $E$. Let $x_{1}, x_{2}, \ldots, x_{q} \in E^{*}$ and assume that $x_{1} \notin F$. Then one of the products

$$
\prod_{j=1, j \neq k}^{q} x_{j}
$$

does not belong to $F$. 
Proof. For each $k=1, \ldots, q$ let

$$
y_{k}=\prod_{j=1, j \neq k}^{q} x_{j}
$$

and suppose that $y_{k} \in F$ for each $k=1, \ldots, q$. First note that none of the $y_{k}$ is zero, because no $x_{j}$ is zero. For each $k=1, \ldots, q$, set $x_{k}=b_{k} x_{1}$. Then $b_{k}=y_{1} / y_{k}$ hence $b_{k}$ belongs to $F$. Now,

$$
y_{1}=\left(x_{1}\right)^{q-1} \prod_{j=2}^{q} b_{j}
$$

and therefore $\left(x_{1}\right)^{q-1}$ belongs to $F$. But $F$ is algebraically closed in $E$, hence $x_{1}$ also belongs to $F$, a contradiction.

Henceforth, we will use the classical Nevanlinna functions:

Notation 3.2. Given a function $f \in \mathcal{M}(\mathcal{D})$ such that $f(0) \neq 0, \infty$, we will denote by $Z(r, f)$ the counting function of zeros of $f$ in $\mathcal{D}$, counting multiplicities, and by $N(r, f)$ the counting function of poles of $f$ in $\mathcal{D}$, counting multiplicities. Finally, we denote by $T(r, f)$ the Nevanlinna characteristic function

$$
\max (Z(r, f)+\log (|f(0)|), N(r, f)) .
$$

For convenience, we will take $\mathcal{D}=d\left(0, R^{-}\right)$without loss of generality. The following theorem is easily seen: for instance it is an immediate corollary of Theorem 2.6.4 [?].

Theorem 3.3. Let $f \in \mathcal{M}(\mathcal{D})$ with $f(0) \neq 0, \infty$. Then $T(r, f)$ is bounded when $r$ tends to $R$ if and only if $f$ belongs to $\mathcal{M}_{b}(\mathcal{D})$. Moreover, $\mathcal{M}_{b}(\mathcal{D})$ is algebraically closed in $\mathcal{M}(\mathcal{D})$.

The following theorem is a particular case of Theorem 3.1.6 [?] or Theorem 1 [?].

Theorem 3.4. Let $c_{1}, \ldots, c_{5} \in K$ be pairwise distinct. Then there exist no pair $(g, f) \in \mathcal{M}_{u}(\mathcal{D})$ such that

$$
f^{2}=\prod_{j=1}^{5}\left(c_{j}-g\right) .
$$

That is, each $\mathcal{M}(\mathcal{D})$-parameterizations of the hyper-elliptic curve

$$
y^{2}=\prod_{j=1}^{5}\left(c_{j}-x\right)
$$

is actually a $\mathcal{M}_{b}(\mathcal{D})$-parametrization.

Theorem ?? allows us to apply Lemma ?? to the field extension $\mathcal{M}(\mathcal{D}) / \mathcal{M}_{b}(\mathcal{D})$ and obtain the next lemma with help of Theorem ??.

Lemma 3.5. Let $M \geq 10$ and let $f, g \in \mathcal{M}(\mathcal{D})$ with $g$ non-identically zero. Let $h_{1}, \ldots, h_{M} \in \mathcal{M}(\mathcal{D})$ and assume that at least one of the $h_{j}$ belongs to $\mathcal{M}_{u}(\mathcal{D})$. Suppose there exist $a_{1}, \ldots, a_{M} \in K$ distinct such that

$$
h_{j}^{2}=\left(a_{j}+f\right)^{2}-g
$$

for each $j=1, \ldots, M$. Then $f$ is not constant. 
Proof. By contradiction, assume that $f$ is constant. Since at least one of the $h_{j}$ is not constant (indeed, at least one of the $\left.h_{j} \notin \mathcal{M}_{b}(\mathcal{D})\right)$ we get that $g$ is non-constant and thus all the $h_{j}^{2}$ are non-constant. In particular no $h_{j}$ is the zero function.

By Theorem ??, $\mathcal{M}_{b}(\mathcal{D})$ is algebraically closed in $\mathcal{M}(\mathcal{D})$. Consequently, by Lemma ??, there are 9 elements in $\left\{h_{1}, \ldots, h_{M}\right\}$ whose product lies in $\mathcal{M}_{u}(\mathcal{D})$. Without loss of generality, we may assume that

$$
\prod_{j=1}^{9} h_{j} \in \mathcal{M}_{u}(\mathcal{D}) \text {. }
$$

Let $\phi=h_{1} \cdots h_{9}$. For each $j=1, \ldots, 9$ let $c_{j}=\left(a_{j}+f\right)^{2} \in K$. Since the $a_{j}$ are pairwise distinct, we can conclude that no three of the $c_{j}$ are equal because $c_{i}=c_{j}$ implies $a_{i}+a_{j}=2 f$. Consequently, among the $\left\{c_{1}, \ldots, c_{9}\right\}$ there are at least 5 pairwise distinct elements, and without loss of generality we assume that $c_{1}, \ldots, c_{5}$ are pairwise distinct. Then we have

$$
\phi^{2}=\prod_{j=1}^{5}\left(c_{j}-g\right) .
$$

Since $\phi$ lies in $\mathcal{M}_{u}(\mathcal{D})$, so does $g$ (because $\mathcal{M}_{b}(\mathcal{D})$ is algebraically closed in $\mathcal{M}(\mathcal{D})$ ). But $(g, \phi)$ gives a parametrization of the hyper-elliptic curve of equation

$$
y^{2}=\prod_{j=1}^{5}\left(c_{j}-x\right) .
$$

which contradicts Theorem ??. This proves that $f$ is not a constant.

We notice that in [?], all claims $1,2,3,4,5$ only use inequalities that hold in $\mathcal{M}(K)$ as well as in $\mathcal{M}(\mathcal{D})$. Consequently their conclusions hold in $\mathcal{M}(\mathcal{D})$.

The following lemma is Claim 2 in [?].

Lemma 3.6. Let $f, g \in \mathcal{M}(\mathcal{D})$ with $g$ non-identically zero. Let $h_{1}, \ldots, h_{M} \in \mathcal{M}(\mathcal{D})$ with $h_{1} \in \mathcal{M}_{u}(\mathcal{D})$ and let $a_{1}, \ldots, a_{M} \in K$ be distinct and such that

$$
h_{j}^{2}=\left(a_{j}+f\right)^{2}-g
$$

for each $j=1, \ldots, M$. Then

$$
\sum_{j=1}^{M} Z\left(r, h_{j}\right) \geq \frac{M-3}{M-1} \sum_{j=1}^{M} N\left(r, h_{j}\right)+O(1) .
$$

\section{Proof of Theorem ??}

In this section, we present the proof of Theorem ??. Suppose $M \geq 35$ in the general case and that $M \geq 13$ when all $h_{j}$ belong to $\mathcal{A}(\mathcal{D})$ with $h_{1} \in \mathcal{A}_{u}(\mathcal{D})$.

Let $\Delta=\left(g^{\prime}\right)^{2}-4\left(f^{\prime}\right)^{2} g$. First, we will show that $\Delta=0$. The process is quite similar to this of Claim 6 in [?] but requires some precisions at the end. Indeed, assume that $\Delta$ is not identically zero. Set

$$
\mathcal{Z}=\sum_{j=1}^{M} Z\left(r, h_{j}\right)
$$

and

$$
\mathcal{N}=\sum_{j=1}^{M} N\left(r, h_{j}\right)
$$


As in the proof of Claim 6, we have

$$
-2 \log r \geq\left(\frac{1}{2}-\frac{6 M-2}{M(M-1)}\right) \mathcal{Z}+\left(\frac{6 M-2}{M(M-1)}-\frac{16}{M-1}-\frac{8}{(M-1)^{2}}\right) \mathcal{N}+O(1)
$$

hence (since $\log r$ is bounded):

$$
O(1) \geq\left(\frac{M^{2}-13 M+4}{2 M(M-1)}\right) \mathcal{Z}+\left(\frac{-10 M^{2}+2}{2 M(M-1)^{2}}\right) \mathcal{N} .
$$

Suppose first that $\mathcal{N}$ is bounded when $r$ tends to $R$, hence $N\left(r, h_{j}\right)$ is bounded for each $j=1, \ldots, M$. Since at least one of the $h_{j}$ belongs to $\mathcal{M}_{u}(\mathcal{D})$, by Theorem ?? there exists at least one index $k$ such that $T\left(r, h_{k}\right)$ is not bounded when $r$ tends to $R$ and hence $Z\left(r, h_{k}\right)$ is not bounded when $r$ tends to $R$. Consequently, $\mathcal{Z}$ cannot be bounded when $r$ tends to $R$. Now, since $M^{2}-13 M+4>0$ because $M \geq 13$,

$$
\left(\frac{M^{2}-13 M+4}{2 M(M-1)}\right) \mathcal{Z}+\left(\frac{-10 M^{2}+2}{2 M(M-1)^{2}}\right) \mathcal{N}
$$

tends to $+\infty$ when $r$ tends to $R$, hence we have a contradiction. Particularly this applies to the case when all $h_{j}$ belong to $\mathcal{A}(\mathcal{D})$ with $h_{1} \in \mathcal{A}(\mathcal{D})$.

Suppose now that $\mathcal{N}$ is not bounded when $r$ tends to $R$. By Lemma ??, we have

$$
\mathcal{Z} \geq \frac{M-3}{M-1} \mathcal{N}
$$

hence

$$
\left(\frac{M^{2}-13 M+4}{2 M(M-1)}\right) \mathcal{Z}+\left(\frac{-10 M^{+} 2}{2 M(M-1)^{2}}\right) \mathcal{N} \geq \frac{M^{3}-36 M^{2}+43 M-8}{2 M(M-1)^{2}} \mathcal{N}
$$

which tends to $+\infty$ when $r$ tends to $R$ because $M \geq 35$. So, we have proved that $\Delta=0$ in the general case whenever $M \geq 35$ and when the $h_{j}$ belong to $\mathcal{A}(\mathcal{D})$, whenever $M \geq 13$. Henceforth, we can assume that $\Delta=0$.

Next, we will follow a similar way as in [?], with however some change. Since $\Delta=0$, we have $\left(g^{\prime}\right)^{2}=4\left(f^{\prime}\right)^{2} g$ and since $f$ is not a constant, this proves that there exists $u \in \mathcal{M}(\mathcal{D})$ such that $u^{2}=g$ and $\left(u^{\prime}\right)^{2}=\left(f^{\prime}\right)^{2}$. Consequently, $u$ is of the form $\epsilon f+b$ with $\epsilon=1$ or -1 (but fixed) and $b \in K$. Therefore, for each $j=1, \ldots, M$ we check that

$$
\left(h_{j}\right)^{2}=\left(a_{j}-\epsilon b\right)\left(a_{j}+\epsilon b+2 f\right) .
$$

Note that for each $j$ the function $a_{j}+\epsilon b+2 f$ is not identically zero by Lemma ??, and note also that $a_{j}-\epsilon b=0$ at most for one $j$, because the $a_{j}$ are pairwise distinct and $\epsilon b$ is fixed. Therefore at least $M-1>5$ of the functions $h_{j}$ are not identically zero, including $h_{1}$ which belongs to $\mathcal{M}_{u}(\mathcal{D})$ by hypothesis.

Now, by Lemma ??, there are 5 elements in $\left\{h_{1}, \ldots, h_{M}\right\}$ whose product lies in $\mathcal{M}_{u}(\mathcal{D})$. Without loss of generality, we may assume (just for notational convenience) that

$$
\prod_{j=1}^{5} h_{j} \in \mathcal{M}_{u}(\mathcal{D})
$$

Let $\phi=\prod_{j=1}^{5} h_{j}$. By Equation (??), we have

$$
\phi(x)^{2}=\prod_{j=1}^{5}\left(a_{j}-\epsilon b\right)\left(a_{j}+\epsilon b+2 f\right) .
$$


But since $\phi$ belongs to $\mathcal{M}_{u}(\mathcal{D})$, so does $f$. Consequently, the hyper-elliptic curve of equation

$$
y^{2}=\prod_{j=1}^{5}\left(a_{j}-\epsilon b\right)\left(a_{j}+\epsilon b+2 x\right)
$$

admits a parametrization by a pair of functions lying in $\mathcal{M}_{u}(\mathcal{D})$, a contradiction to Theorem ??. This finishes proving that $M \leq 34$ and that $M \leq 12$ when all $h_{j}$ belong to $\mathcal{A}_{u}(\mathcal{D})$.

\section{References}

[1] Boutabaa A. and Escassut, A. URS and URSIMS for p-adic meromorphic functions inside a disk. Proc. Edinb. Math. Soc., N 44; p. 485-504 (2001).

[2] Escassut, A. p-adic Value Distribution. Some Topics on Value Distribution and Differentability in Complex and P-adic Analysis, p.42- 138. Mathematics Monograph, Series 11. Science Press.(Beijing 2008)

[3] Pasten H. An analogue of Büchi's Problem for p-adic meromorphic functions, to appear in Transactions of the AMS.

[4] T. Pheidas and X. Vidaux The analogue of Büchi's problem for rational functions, Journal of The London Mathematical Society 74-3, 545-565 (2006).

[5] T. Pheidas and X. Vidaux Corrigendum: The analogue of Büchi's problem for rational functions, Journal of The London Mathematical Society, to appear in the Journal of the London Mathematical Society.

[6] Vojta, P.Diagonal quadratic forms and Hilbert's tenth problem, pp. 261-274 in Hilbert's tenth problem: relations with arithmetic and algebraic geometry (Ghent, 1999), Contemp. Math. 270, Amer. Math. Soc., Providence, RI, 2000.

Alain Escassut

Laboratoire de Mathematiques UMR 6620

Université Blaise Pascal

Les Cézeaux

63171 AUBIERE CEDEX

FRANCE

Hector Pasten

Math Department at Queen's University,

KINGSTON

ON, K7L 3N6.

CANADA 